\title{
Endoscopic treatment of walled-off pancreatic necrosis by simultaneous transgastric and retroperitoneal approaches
}

Infected walled-off pancreatic necrosis (WOPN) is a severe complication of acute pancreatitis. In cases of extensive WOPN developing away from the stomach wall, endoscopic treatment may not be effective [1]. A complementary treatment by percutaneous drainage can be performed, allowing retroperitoneal access $[2,3]$. We report the case of a large and complicated infected WOPN, successfully treated by an innovative double endoscopic necrosectomy approach, associating simultaneous transgastric and retroperitoneal endoscopic necrosectomy.

A 72-year-old man presented with severe acute biliary pancreatitis. He developed a symptomatic infected WOPN, mainly lo-
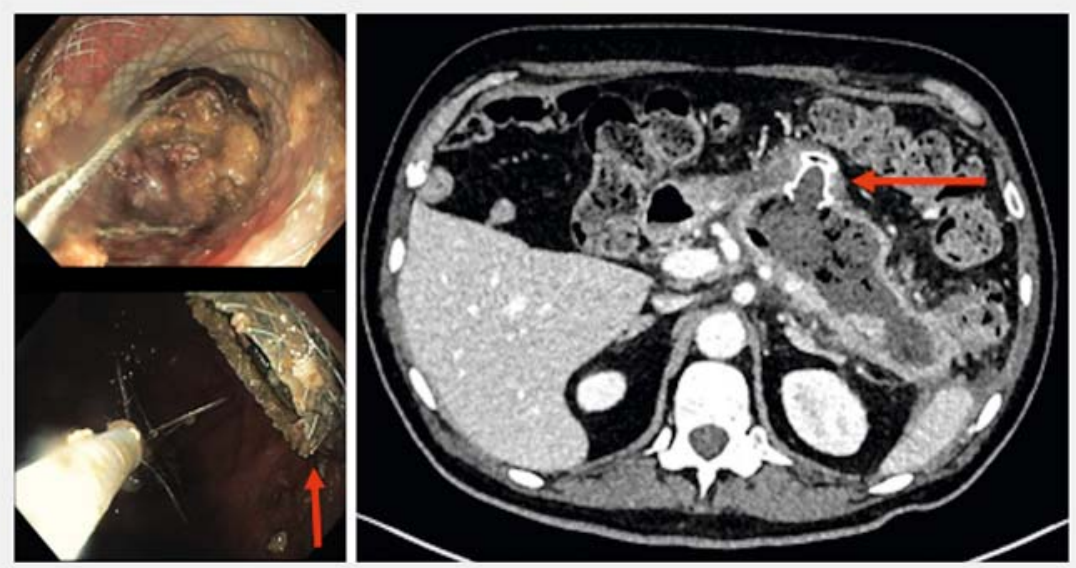

- Fig. 1 Endoscopic necrosectomy sessions by transgastric drainage with a lumen-apposing metal stent (red arrow).
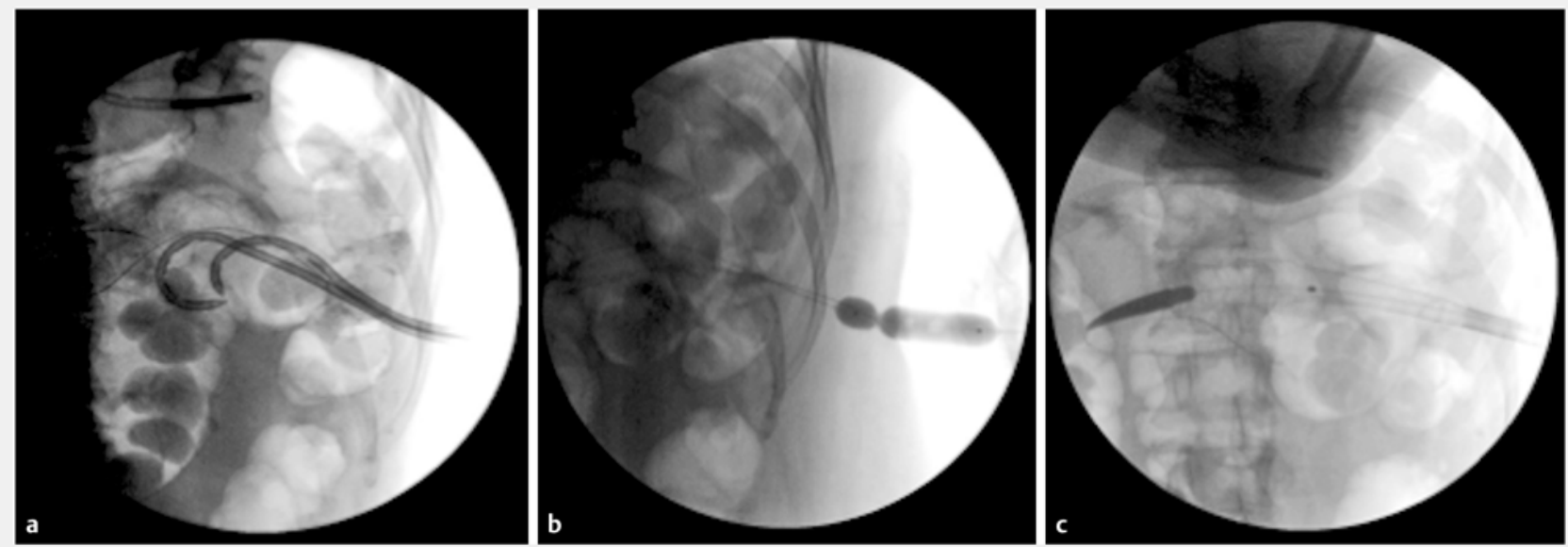

Fig. 2 Percutaneous drainage of the walled-off pancreatic necrosis. a Previous percutaneous drainage by two 10-Fr radiologic drains. $\mathbf{b}$ Dilation of the fistula with a hydrostatic balloon. $\mathbf{c}$ A wide-bore fistula was created by an esophageal, partially covered, self-expandable metal stent. 


\section{Endoscopic treatment of walled-off pancreatic necrosis by simultaneous transgastric and retroperitoneal approaches}

Enrique Pérez-Cuadrado-Robles ${ }^{1}$, Arthur Berger ${ }^{1}$, Guillaume Perrod ${ }^{1}$, Emilia Ragot ${ }^{2}$, Charles André Cuenod ${ }^{3}$, Gabriel Rahmi ${ }^{1}$, Christophe Cellier ${ }^{1}$.

Department of Gastroenterology ${ }^{1}$, Sugery ${ }^{2}$ and Radiology ${ }^{3}$. GeorgesPompidou European Hospital. Paris, France.

Video 1 Simultaneous transgastric and retroperitoneal endoscopic treatment of walled-off pancreatic necrosis.

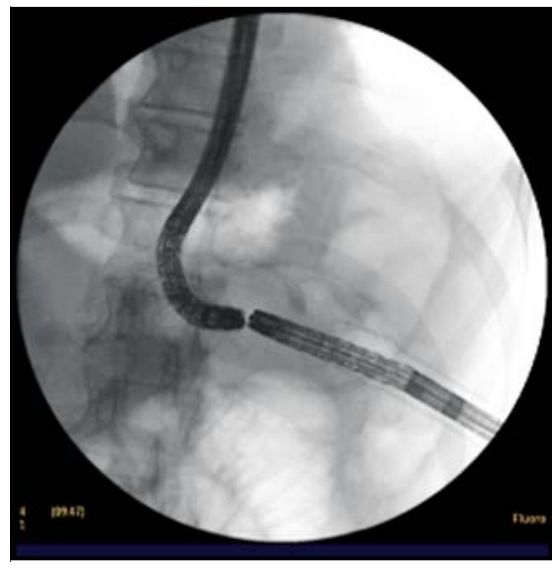

- Fig. 3 Double endoscopic approach by transgastric and percutaneous access.

Once created, the fistula allowed multidisciplinary techniques such as insertion of surgical clamps under endoscopic guidance, and simultaneous endoscopic necrosectomy through both percutaneous and transgastric access ( $\triangleright$ Fig. 3 , - Video 1).

Complete resolution of the WOPN was obtained after two sessions. Between the two procedures, the esophageal patient recovered completely.

Competing interests

None

The authors Christophe Cellier ${ }^{1}$ European Hospital, Paris, France

3 Department of Radiology, Georges
Corresponding author

\section{Arthur Berger, MD, PhD}

Department of Gastroenterology, Georges Pompidou European Hospital, 20 rue Leblanc, 75015 Paris, France

Fax: +33-1-56092914

arthur.berger.bx@gmail.com

References

[1] Bang JY, Arnoletti JP, Holt BA et al. An endoscopic transluminal approach, compared with minimally invasive surgery, reduces complications and costs for patients with necrotizing pancreatitis. Gastroenterology 2019; 156: 1027 - 1040

[2] Laopeamthong I, Tonozuka R, Kojima H et al. Percutaneous endoscopic necrosectomy using a fully covered self-expandable metal stent in severe necrotizing pancreatitis. Endoscopy 2019; 51: E22 -E23

[3] Seifert H, Wehrmann T, Schmitt T et al. Retroperitoneal endoscopic debridement for infected peripancreatic necrosis. Lancet 2000; 356: $653-655$ placed and removed progressively to allow fistula healing. The enterocutaneous fistula was closed after 2 weeks and the

Endoscopy_UCTN_Code_TTT_1AR_2AI

\section{Bibliography}

DOI https://doi.org/10.1055/a-1011-3555

Published online: 27.9.2019

Endoscopy 2020; 52: E88-E89

(c) Georg Thieme Verlag KG

Stuttgart · New York

ISSN 0013-726X

Enrique Pérez-Cuadrado-Robles ${ }^{1}$, Arthur Berger $^{1}$, Guillaume Perrod ${ }^{1}$, Emilia Ragot ${ }^{2}$, Charles André Cuenod ${ }^{3}$, Gabriel Rahmi ${ }^{1}$,

1 Department of Gastroenterology, Georges Pompidou European Hospital, Paris, France

2 Department of Surgery, Georges Pompidou Pompidou European Hospital, Paris, France

\section{ENDOSCOPY E-VIDEOS}

https://eref.thieme.de/e-videos

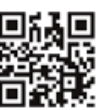
Endoscopy E-Videos is a free access online section, reporting on interesting cases and new techniques in gastroenterological endoscopy. All papers include a high quality video and all contributions are freely accessible online.

This section has its own submission website at

https://mc.manuscriptcentral.com/e-videos 